\title{
Zoom data analysis in an introductory course in mechanical engineering
}

\section{Hong Tao, Bohan Feng}

Department of Mechanical and Aerospace Engineering, The Hong Kong University of Science and Technology, Hong Kong, China.

\begin{abstract}
This investigation studies various data extracted from Zoom meetings which are used in teaching an introductory course in mechanical engineering online. The effect of using Chat tool is discussed by extracting and analyzing the data in chat report. The parameters, such as number of chatting students, chatting participation rate, as well as average numer of chats per student, can show students activeness from different perspectives. Participcation in polling and polling performance are also studied by extracting data from polling reports. Non-graded polling activities are found to be effective of increase attendance of students. The using of breakout room doesn't show its effectiveness in online learning of this introductory course.
\end{abstract}

Keywords: Online learning; engagement; attentiveness; engineering course. 


\section{Introduction}

Creating a good learning atmosphere is one of the key skillsets of instructors. Currently there is no standard definition what is a good learning atmosphere. But one of the key characteristics of a good learning atmosphere is that the class is highly participatory and students are greatly inspired and are actively engaged in the class activities. Students engagement and attentiveness are believed to play a critical role in active and effective learnings. Enhancing students engagement has been extensively researched in higher education in diverse environment and various strategies have been developed. Some research studies are conducted at institutional level and conclusions are drawn from institutional perspective. Kuh (et al 2005) finds institutions that accommodate diverse students learning styles tend to ensure higher engagement of students. Some studies evaluate the effect of different pedagogical technique on students engagement. Gao et al (2020) specifically find the playfulness and usefulness of blended learning platform positively impact on students participation and engagement. Bond (2020) particularly find flipped learning involving collaborative peer teaching and learning positively affect students engagement. Other investigations focus on certain courses in specific fields. For example, Pfenig (2020) finds lecture videos are beneficial to students concentration; Garcia and Valle (2020) suggest that collaborative tasks in virtural classroom increase students engagement. Wu et al (2020) described how drawing prompts have helped engaging students in an introductory engineering course.

Although online courses are increasingly adopted in many countries in recent years, online teaching and learning are still in the developing stage and faced with many challenges. Dynarski (2018) claims online courses are beneficial to academically well prepared and strongly self-motivated students, but are actually harmful to students who are faced with academic challenges. It is worthwhile to further study and develop effective methods of engaging students in online courses.

Since the outbreak of COVID-19 in beginning of 2020, majority of undergraduate courses are shifted to online learning. First year undergraduate students in Fall 2020 have faced double challenges. On one hand, they have experienced the transition from being high-school students to college students and adapted to new learning environment and new ways of college life in their first semester. On the other hand, they also have to cope with unique challenges imposed by outbreak of COVID-19 and online learning in almost all courses.

This paper shares how we have coped with these challenges in teaching first year undergraduate students an introductory course in mechanical engineering and what lessons we have learnt and what further approaches can be implemented to improve teaching and learning online. Particularly, we have studied the effect of various ZOOM tools on engaging students during lectures. 


\section{Data Analysis and Discussions}

First year undergraduate course - Introduction to Mechanical Engineering for Modern Life - is a common core course which is intended to give a comprehensive picture of mechanical engineering and describe the contents of its multiple sub-fields, such as mechanics and materials; thermo-fluids; design and manufacturing, as well as aerospace engineering. This course is designed to offer first year undergraduate students a snapshot what impacts mechanical engineering has on modern life and what mechanical engineers do. Due to the outbreak of COVID-19, this course was conducted via ZOOM 5.3.1 (52879.0927) in Fall semester of 2020. At the time of registering this course, whole class is automatically divided into L1 and L2 session in order to achieve effective teaching and learning.

\subsection{Using Chat for discussions in ZOOM}

In face-to-face classes conducted inside a classroom, it often takes tremendous courage and confidence for students to ask questions and to speak in front of their peers, partly because this will have to interrupt the instructor and disturb the flow of lecture. Often only those who are not shy and with great confidence will do. Since classes are shifted virtually online via ZOOM, there is an advantageously effective tool - "Chat" - for students to speak, voice out feedback, express views, and have discussions among their peers. Communication through Chat tool serves as a channel that break the barrier of cold computer screen and bring students and instructors closer.

For each lecture, ZOOM records all chat contents in a text chat report file. From the downloaded chat reports, we have summarized the number of students who used chat to communicate and also have counted the number of "effective" chats. By excluding the number of chats for greeting purposes (e.g. good morning, thank you, etc.), we only counted the "effective" chats which are true communications, such as comments and questions related to course contents during the lecture.

Figure 1 shows the number of chatting students versus the number of attendants for lectures during Sept 8 to Oct 20, 2020. We have seen an obvious linear trend, i.e. when more students attend the lecture, more tend to chat during lectures. It is interesting to find that, for the same number of attendants, L1 session tends to have more students using Chat tool than L2 session. This trend shown in Figure 1 agrees with my observation during lectures that students in L1 session are more active than those in L2 session. Therefore, Figure 1 number of chatting students versus number of attendants can be used as a tool to assess the students activeness. 


\section{Number of chatting students versus number of attendants}

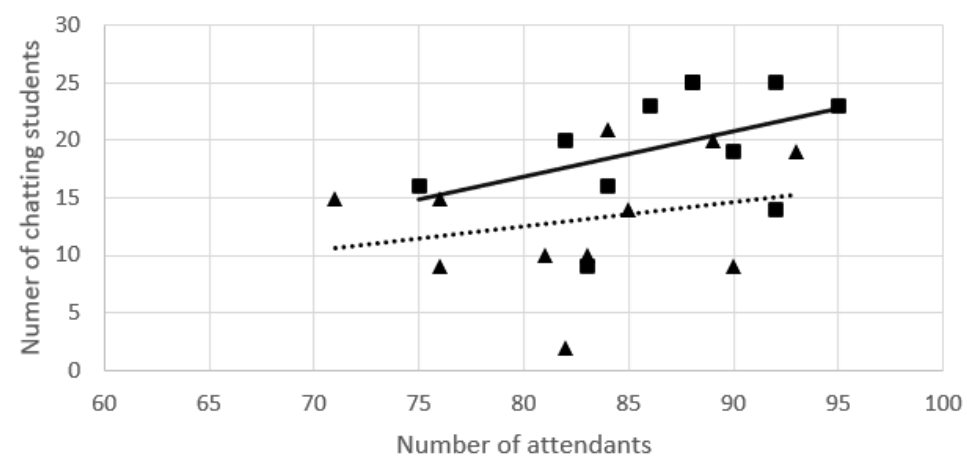

- L1 Session $\Delta$ L2 Session —Linear (L1 Session) ….... Linear (L2 Session)

Figure 1. Number of chatting students versus number of attendants in lectures during Sept 8 to Oct 20, 2020.

Figure 2 shows the chatting participation rate versus lecture dates during Sept 8 to Oct 20, 2020. Chatting participation rate is defined as the ratio of the number of students who chatted to the number of attendants in a lecture. The linear fit shows that, when lecture proceeds with time, the chatting participation rate in L1 session gradually decrease, while the rate of L2 session gradually increases. Chatting participation rate, from a different perspective, can be used as an indicator as the trend or dyanmics of students activeness versus time.

\section{Chatting participation rate}

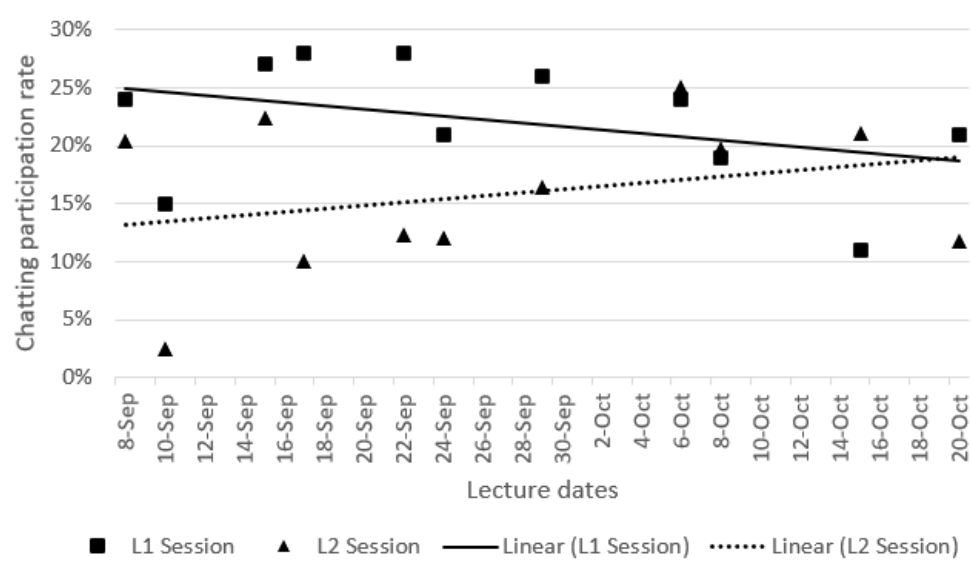

Figure 2. Chatting participation rate versus lecture dates during Sept 8 to Oct 20, 2020.

Figure 3 shows average number of chats per students versus lecture dates during Sept 8 to Oct 20, 2020. It is clearly seen that, in average, each student participating in chat in L2 session chats more than their peers in L1 session. This interesting finding shows that, while more 
students in L1 session use Chat tool than those in L2 session (Figure 1), each chatting students in L2 session chats more (Figure 3). Therefore, the data in Figure 1 and 3 indicate that we can characterize students "activeness" from different perspective, i.e. either by using number of chatting students (i.e. Figure 1) or using average number of chats per chatting student (i.e. Figure 3). They may show different trend.

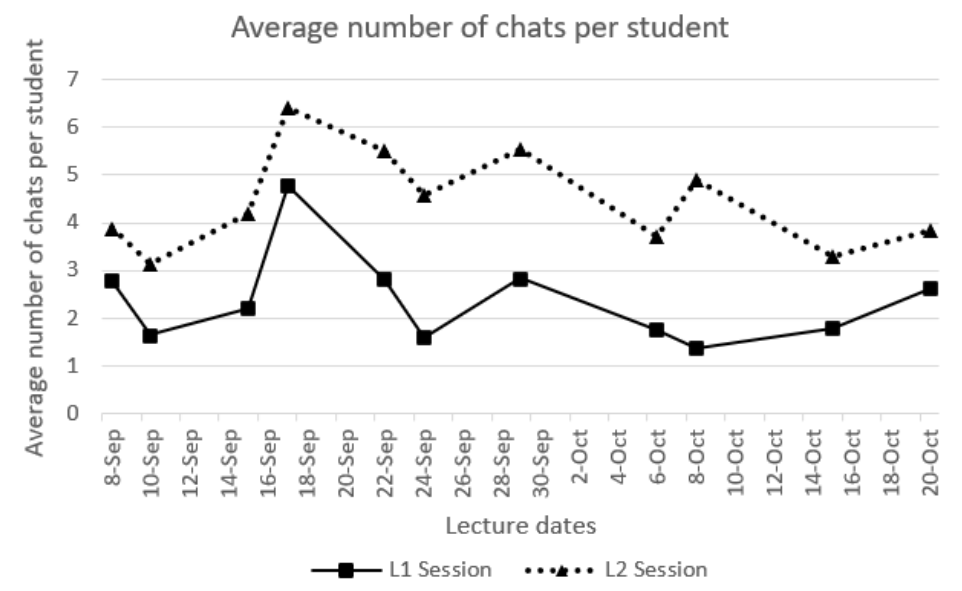

Figure 3. Average number of chats per student in lectures during Sept 8 to Oct 20, 2020.

In this introductory course, the first year undergraduate students are highly active in "Chat". It is suggested that certain class rules may be formulated to regulate the chatting and teaching assistants may be arranged to assist in answering the questions asked in Chat.

\subsection{Using breakout room for small group discussions}

In this introductory course, engineering ethics is a topic that does not involve any calculations and also a topic that may easily hypnotize students on the other side of the computer by teacher's monologue. Breakout rooms are employed and students are placed into each breakout room randomly. It turns out that breakout rooms are not effective for all groups. Some breakout rooms are very quiet and need an ice breaker, some breakout rooms are more active in discussions. It may be partly due to the fact that these first year undergraduate students have not met face-to-face and got to know each other well. How to use breakout rooms effectively in this course needs further investigations.

\subsection{Using polling during lectures}

Zoom has a tool - Polling, which allows users to input questions in the format of either single choice or multiple choices. It is intended to collect attendants' responses to polling questions. In this introductory course, Polling is used as evaluation of students' learning outcomes based on four questions representing four key points of each lecture. Polling evaluation results are not counted into students' grades. For each polling activity, Zoom generates a polling report 
that summarizes the name of the participants as well as their answers to each polling question. Data extracted from the polling report include number of polling participants, participation rate, and performance (i.e. how many answers are correct) of participants in each polling activity.

Figure 4 shows the number of attendants for the lecture dates between September 8 to December 3 in 2020. Each color represents a lecture date. From September 15 to October 20, at the end of each lecture, we launched polling questions as in-class quizzes, but the results of quizzes are not counted to students' final grades. It is obvious that the number of attendants in the lectures with pollings is significantly higher than those without pollings. The average number of attendants for lectures with pollings is 85 , while the average of number of attendants for lectures without pollings is 62. Therefore, polling is helpful to have more attendants in class. Since the polling activities are not graded, then the reason of more students attending lectures with polling may be due to the face that polling can improve their learning.

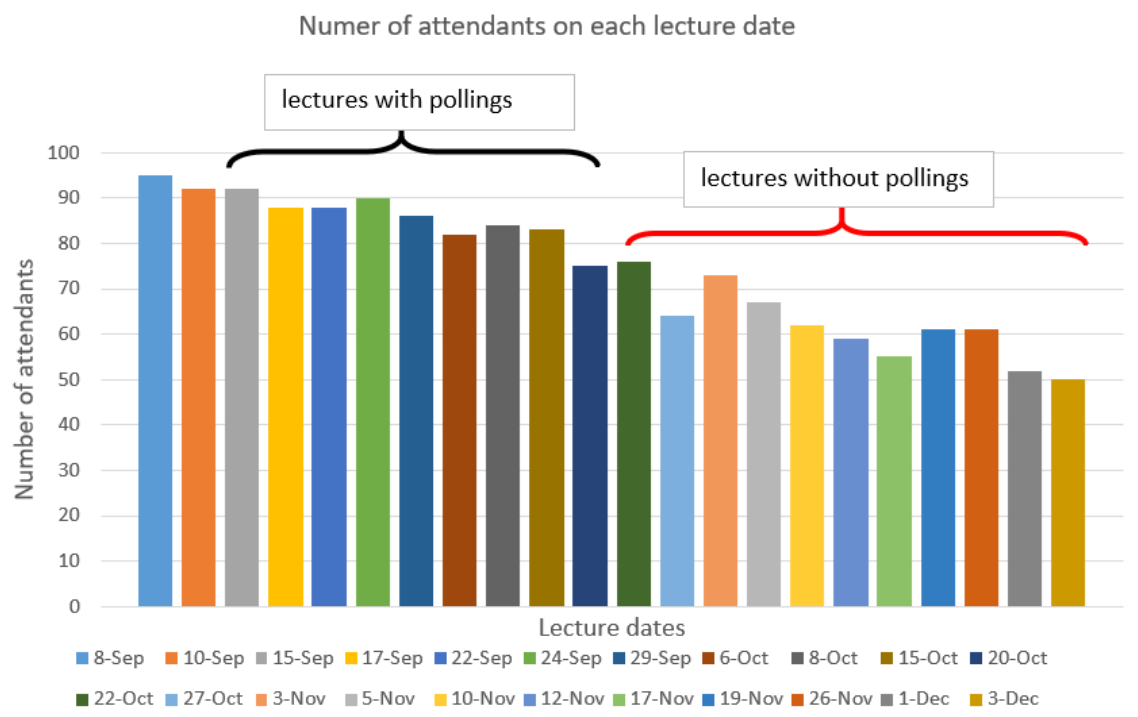

Figure 4. Number of attendants for each lecture date.

Polling participation rate is calculated as the percentage of the number of polliing participants out of the total number of attendants for a specific lecture. Figure 5 shows the polling participation rate versus lecture dates and demonstrates the range between $78 \%$ and $99 \%$. L1 session exhibits slightly declining participation rate, while L2 session has a steady participation rate. It is clearly seen that polling can be used as effective tool to engage students in the in-class activites. 
Polling performance is calculated as the percentage of participants (with $100 \%$ and $75 \%$ correct answers) out of the total number of polling participants in each polling. Figure 6 shows the polling performance (ranging between $50 \%$ to $87 \%$ ) versus lecture dates of L1 and L2 session, respectively. It is interesting to note that L2 session demonstrates a more pronounced increasing trend in polling performance than L1 session.

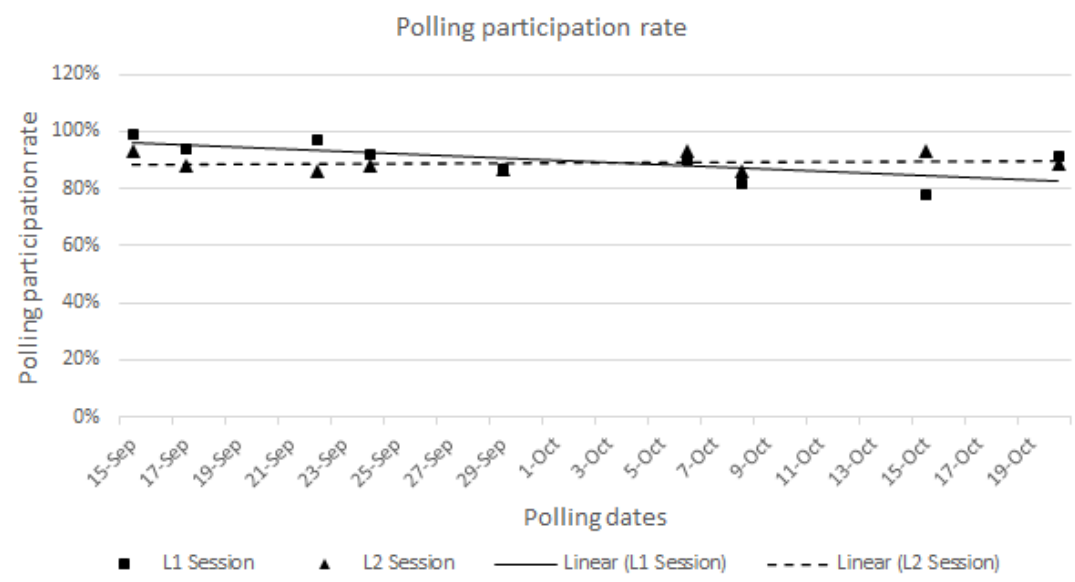

Figure 5. Polling participation rate on lecture dates during September 15 to October 20, 2020.

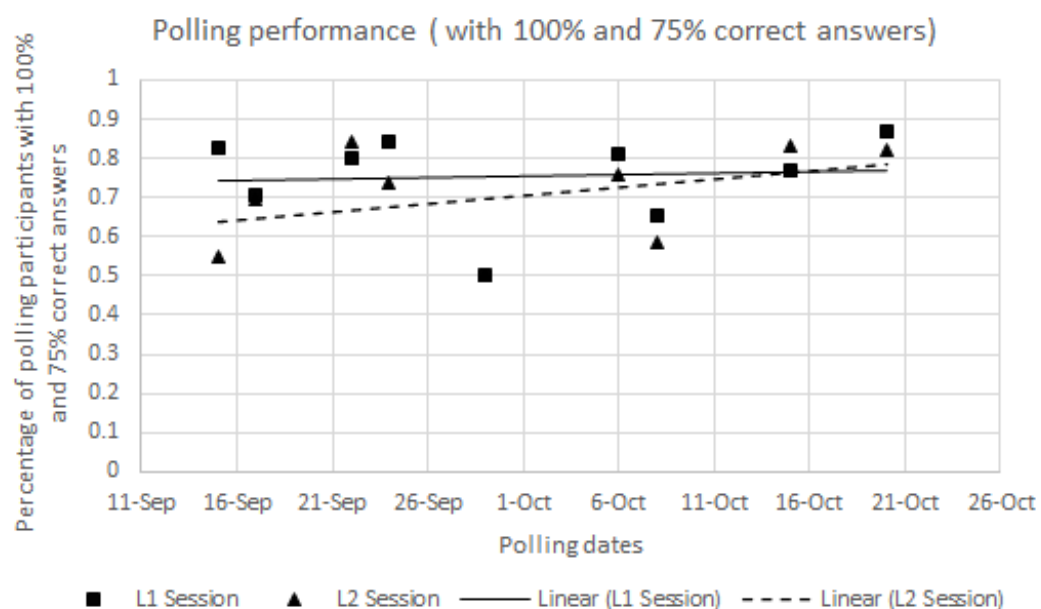

Figure 6. Polling performance (with $100 \%$ and $75 \%$ correct answers) on selected lecture dates. 


\section{Conclusions}

The Chat tool in ZOOM makes boundaries imposed by the cold computer screen dissppear and is very effective for students to communicate with instructors and to discuss course questions among themselves during lecture. The data, i.e. number of students who use Chat, chat participation rate, as well as average chats per student, extracted from the Chat reports, can serve as an indicator for students activeness from differenct perspectives. For classes with very active students such as first year undergraduate students, arranging teaching assistants to help answer questions in chat will partially relieve instructors work load and ensure instructors enough time concentrating on lecture delivery. Polling is another useful tool in Zoom meetings and can be used as in-class quiz tool. The data extracted from polling report can assess students participation and also learning outcome.

\section{References}

Bond, M. (2020) Facilitating student engagement through the flipped learning approach in K-12: A systematic review, Computers \& Education, Volume 151, DOI: https://doi.org/10.1016/j.compedu.2020.103819

Dynarski, S. (2018, Jan 21). Online courses are harming the students who need most help. The New York Times, page BU3.

Gao, B.W., Jiang, J., and Tang, Y. (2020). The effect of blended learning platform and engagement on students' satisfaction - the case from the tourism management teaching. Journal of Hospitality, Leisure, Sport \& Tourism Education, Volume 27, 723-742. DOI: https://doi.org/10.1016/j.jhlste.2020.100272

Garcia, M. A., Valle, A. M. (2020). On strategies to improve students engagement. 6th International Conference on Higher Education Advances (HEAd'20), 1085-1092. DOI: http://dx.doi.org/10.4995/HEAd20.2020.11201

Kuh, G. D., Kinzie, J., Schuh, J. H., and Whitt, E. J. (2005). Student success in college: Creating conditions that matter. Associates American Association. Jossey-Bass.

Pfenig, A. (2020). Lessions learnt - The role of peer - to - peer lecture films in a first year material science laboratory course. 6th International Conference on Higher Education Advances (HEAd'20), 9-16. DOI: http://dx.doi.org/10.4995/HEAd20.2020.10953

Wu, S.P.W., Van Veen, B., and Rau, M.A. (2020). How drawing prompts can increase cognitive engagement in an active learning engineering course. Journal of Engineering Education, 109 (4), 723-742. DOI: https://doi.org/10.1002/jee.20354 